\title{
Undercoolability of Superalloys and Solidification Defects in Single Crystal Components
}

\author{
Dexin $\mathrm{Ma}^{1, \mathrm{a}}$, Qiang $\mathrm{Wu}^{1, \mathrm{~b}}(\mathrm{Sp})$, Andreas Bührig-Polaczek ${ }^{1, \mathrm{c}}$ \\ ${ }^{1}$ Foundry Institute of RWTH Aachen University, Intzestrasse 5, 52072 Aachen, Germany \\ ad.ma@gi.rwth-aachen.de, bq.wu@gi.rwth-aachen.de, 'sekretariat@gi.rwth-aachen.de
}

Key words: superalloy, undercoolability, single crystal, stray grain

\begin{abstract}
The undercooling behaviour of the superalloys IN 939 and CMSX-6 during the isothermal heating and cooling processes was investigated. Under the same process conditions, the undercoolability of CMSX-6 was measured to be $50.4 \mathrm{~K}$ while that of IN 939 was only $10.1 \mathrm{~K}$. This great difference can be attributed to the amount of alloying elements. During Bridgman directional solidification using the same process parameters, the used alloys exhibited a very different tendency to form structure defects. While the CMSX-6 components were nearly defect-free, the IN 939 samples of the same geometry were very prone to stray grain formation. This difference can be attributed to the different undercoolability of the investigated superalloys. Therefore, the undercoolability is an important factor of castability and the cast quality of single crystal components can be effectively improved by increasing the undercoolability of the superalloys.
\end{abstract}

\section{Introduction}

In order to enhance the efficiency of stationary gas turbines, the demand for larger single crystal (SC) castings has dramatically increased [1]. In recent years, the established technology for aeroengines has been transferred to stationary gas turbines. However, despite efforts made over a number of years, the control of the industrial casting process remains unsatisfactory; resulting in a significant number of the components produced being rejected due to solidification defects. The most serious solidification defects are those which feature a high-angle boundary. These boundaries can be due to a number of different causes. For example, the extremities of the platform cool more rapidly than the blade body. As a result, an isolated, thermally undercooled region of melt is formed. When the local undercooling is sufficient to exceed the undercoolability of the used alloy, new grains may nucleate to generate macroscopic stray grain defects $[2,3]$.

Undercooling plays an important role in determining the microstructure development and phase selection because nucleation and subsequent crystal growth govern the microstructure and phase formation. The directional solidification (DS) of an undercooled melt was proposed by Lux et al.[4]. Correspondingly, an autonomous directional solidification (ADS) processing [5] was developed, in which the superalloys were undercooled in a suitable ceramic shell mould system to a certain degree but not exceeding the undercoolability, thus leading to a DS process after nucleation at the bottom of the mould. According to Lux et al., different superalloys have different values of undercoolability. The factors influencing the undercoolability are the chemical composition of the alloys and the process conditions such as the shell mould system, the process atmosphere (vacuum or inert gas) and the thermal conditions (overheating, cooling rate etc.).

In our research, the undercoolability of superalloys was investigated under the same process conditions, in order to distinguish the chemical composition dependence of the undercoolability. The simplified turbine blades were also produced using Bridgman process to determine the dependence of the solidification defects on the undercoolability. 


\section{Materials and experimental procedures}

\subsection{Materials}

The commercial superalloys of IN 939 and CMSX-6 (Table 1) were chosen to investigate the undercoolability of superalloys under the same process conditions and to determine the dependence of the solidification defects on the undercoolability in Bridgman process.

Table 1. Nominal composition of the used superalloys [wt.\%]

\begin{tabular}{cccccccccccc}
\hline & $\mathrm{Cr}$ & $\mathrm{Co}$ & $\mathrm{W}$ & $\mathrm{Mo}$ & $\mathrm{Al}$ & $\mathrm{Ti}$ & $\mathrm{Ta}$ & $\mathrm{Zr}$ & $\mathrm{B}$ & $\mathrm{C}$ & $\mathrm{Ni}$ \\
\hline IN 939 & 22.4 & 19.0 & 2.0 & - & 1.9 & 3.7 & 1.4 & 0.10 & 0.009 & 0.15 & bal \\
CMSX-6 & 10.0 & 5.0 & - & 3.0 & 4.8 & 4.7 & 2.0 & - & - & - & bal \\
\hline
\end{tabular}

\subsection{Measurement of the undercooling}

The undercoolability $\left(\Delta \mathrm{T}_{\mathrm{a}}\right)$ of an alloy is its ability to be cooled to a temperature below the melting point $\left(\mathrm{T}_{\mathrm{L}}\right)$ without onset of solidification. The undercoolability can be determined as the statistic average of the critical nucleation undercooling $\left(\Delta \mathrm{T}_{\mathrm{N}}\right)$ measured during a vast amount of experiments, because different $\Delta \mathrm{T}_{\mathrm{N}}$-values can be measured even though the experimental condition remains the same. In order to determine the undercoolability of both superalloys under the same process condition, a shell mould cluster was designed to measure the undercooling, as shown in Fig. 1. For this purpose, a wax model with two separated segments was assembled, each of which concluded five small spherical samples of $12 \mathrm{~mm}$ in diameter. The feed-in gate was also divided into two corresponding independent parts. Therefore, both alloys could be heated and cooled simultaneously but individually.

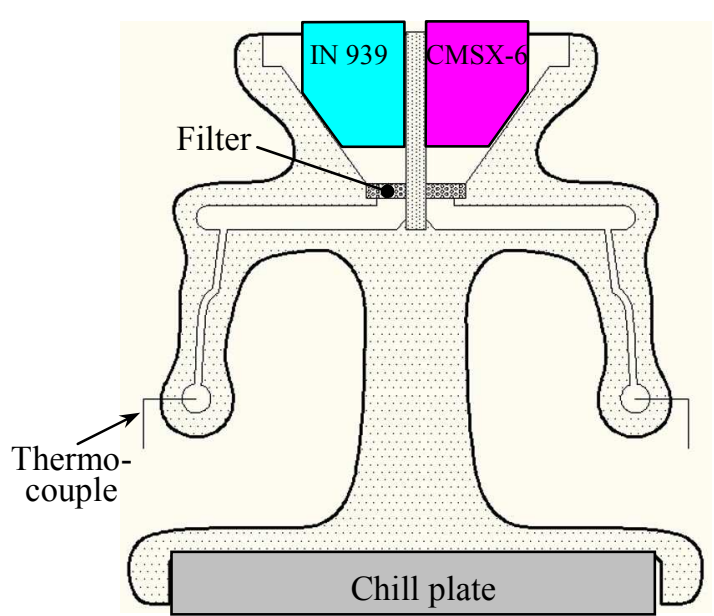

(a)

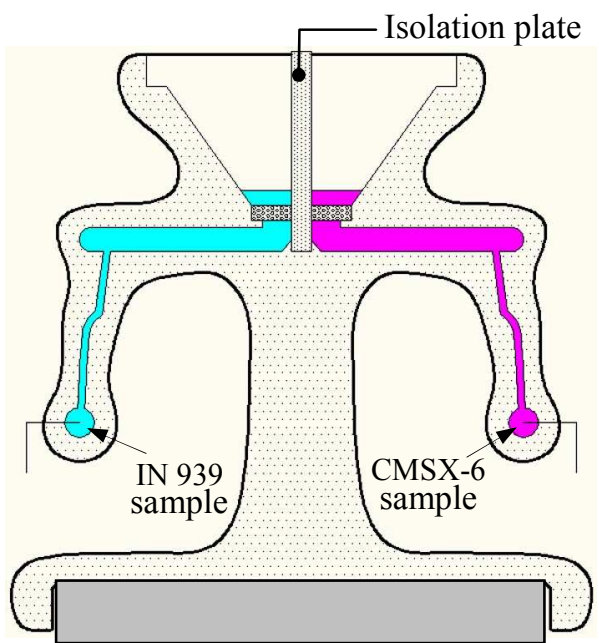

(b)

Fig.1. Schematics of the shell mould to simultaneously investigate the undercooling behaviour of IN 939 and CMSX-6. (a) Prior to the melting of the materials; (b) After the melting and filling into the spherical cavities.

The ceramic moulds of $\mathrm{Al}_{2} \mathrm{O}_{3}$ were manufactured by standard investment casting procedure. To measure the temperature development in the samples during the process, the thermocouples of type "B" (Pt-30\%Rh/Pt-6\%Rh) were installed into the ceramic sheaths in the shell mould. Prior to the experiment the shell mould was placed in a vacuum furnace and the separated sprues were filled with alloy materials with a weight of about $250 \mathrm{~g}$. The procedure of the melting-solidification cycle is illustrated in the Fig. 2. After evacuation, the furnace was heated to a heater temperature $\mathrm{T}_{\mathrm{H}}$ of $1500^{\circ} \mathrm{C}$. In this heating process the alloys were melted individually and filled into the designed spherical cavities. After a homogenizing time of 10 minutes, the heaters were switched off to cool 
down the melt, and during this cooling period the melt solidified. When the heater temperature dropped to $1220^{\circ} \mathrm{C}$, the heaters were switched on again to start a new melting-solidification cycle, thus making it possible to obtain more experimental data. During the heating and cooling processes, namely the melting-solidification cycles, the temperature evolutions of the samples were recorded.

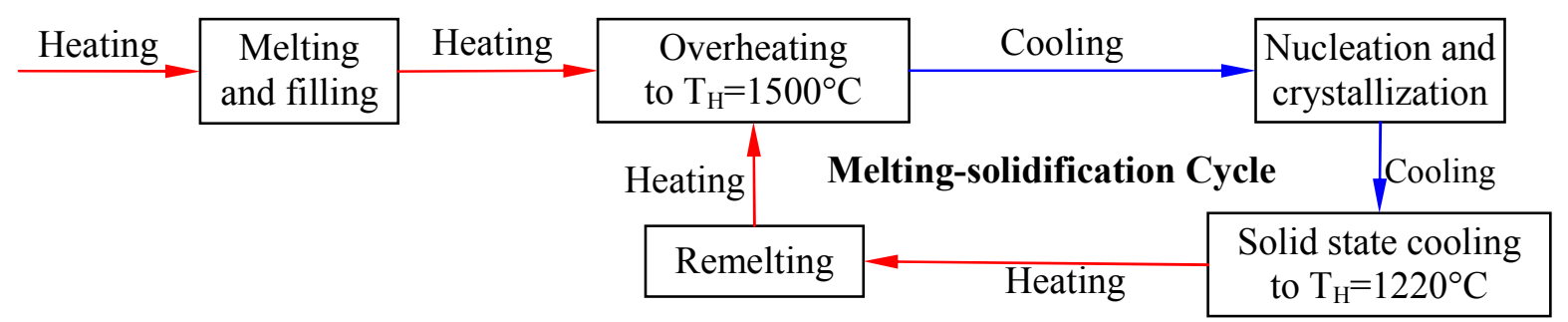

Fig.2. Flowchart of melting-solidification cycle of the undercooling experiment

\subsection{DS using Bridgman process}

For the Bridgman experiments two types of component geometry were designed, providing a simplified geometric feature of the turbine blades (Fig.3a and b). The projection of the platform from the blade body is $16 \mathrm{~mm}$ in Type A and $10 \mathrm{~mm}$ in Type B. Since grain defects occur particularly at the abrupt transition of the cross-section, three platforms were designed in each blade in order to investigate the formation of stray grain more effectively. (Actual turbine blades only feature one or two platforms.) Fig.3c shows the corresponding shell mould of the cluster, with 8 components, 4 pieces of geometry A and 4 pieces of geometry B respectively, symmetrically assembled around a central rod. Two shell moulds were produced for the DS experiment of IN 939 and CMSX-6, respectively.

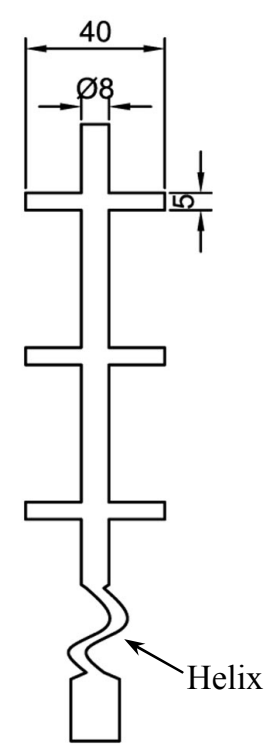

(a) Geometry A

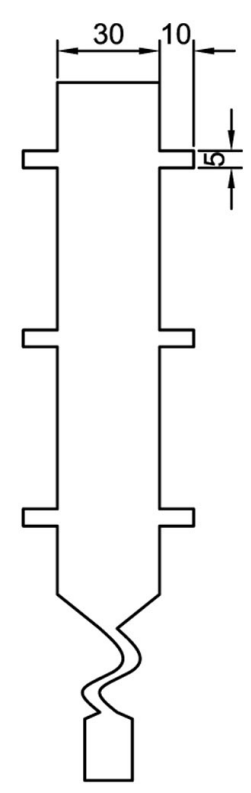

(b) Geometry B

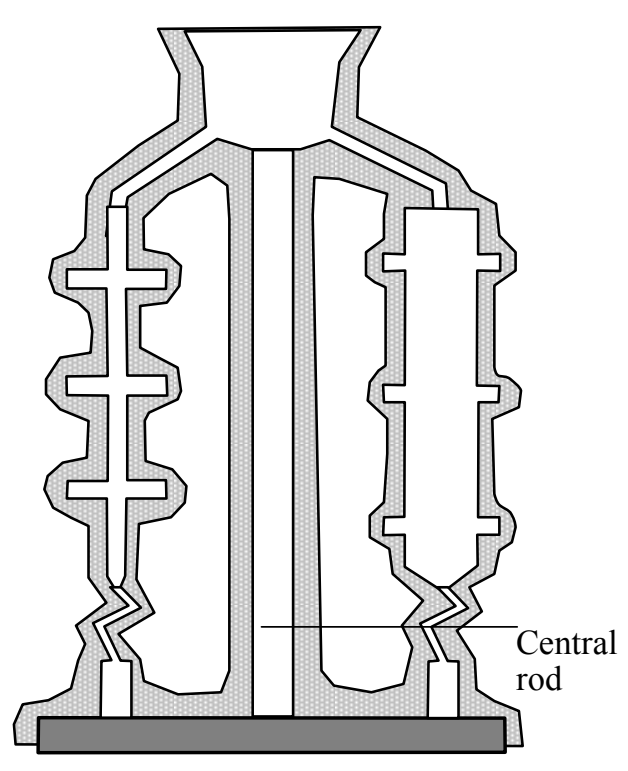

(c) Shell mould

Fig.3. Dimensions of the simplified turbine blades $(a, b)$ and illustration of shell mould cluster (c).

The casting of the two shell moulds, with IN 939 and CMSX-6 respectively, was carried out using the same process parameters. The pouring and heater temperatures were $1500^{\circ} \mathrm{C}$. After preheating the shell mould and melting the alloy under investigation, the liquid metal was poured into the shell mould on the chill plate. The chill mould was then withdrawn from the hot zone to the cold zone through the baffle at a velocity of $3.0 \mathrm{~mm} / \mathrm{min}$. The casting samples were inspected by grain etching with $\mathrm{HCl}+\mathrm{H}_{2} \mathrm{O}_{2}$. 


\section{Results and discussion}

\subsection{Determination of undercoolability}

A typical temperature curve is shown in Fig.4. The nucleation temperature $T_{N}$ and the liquidus temperature $\mathrm{T}_{\mathrm{L}}$ could be evaluated from the cooling and heating curves respectively. The undercooling for the onset of solidification in this cycle is then $\Delta T_{N}=T_{L}-T_{N}$. The average value of the measured undercooling of each alloy is defined as the undercoolability $\Delta \mathrm{T}_{\mathrm{a}}$ of the alloy under the corresponding process condition.

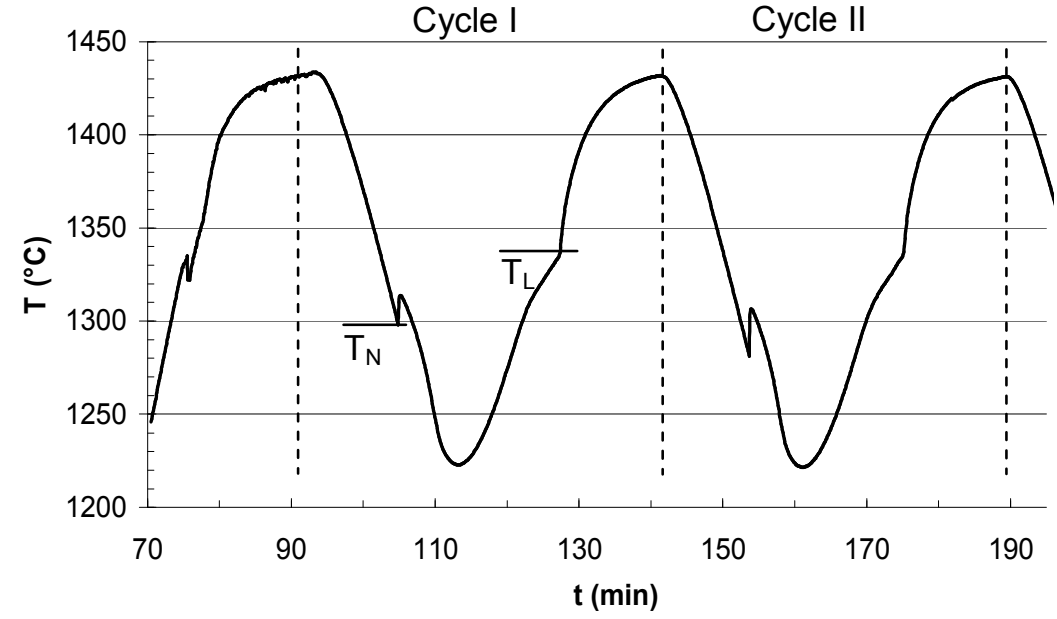

Fig.4. A typical heating and cooling curve of a CMSX-6 sample measured in the meltingsolidification cycles, revealing the nucleation temperature $\mathrm{T}_{\mathrm{N}}$ and liquidus temperature $T_{L}$.

Table 2 summarizes the measurement data of all samples during the two applied meltingsolidification cycles. The nucleation undercooling of CMSX6 was measured to be between 76.0 and $32.0 \mathrm{~K}$, while that of IN939 is only between 17.2 and $5.5 \mathrm{~K}$. As an average of the undercooling value, the undercoolability $\Delta \mathrm{T}_{\mathrm{a}}$ was also evaluated for both used alloys. As shown in Table 2, the undercoolability of CMSX6 $\left(\Delta \mathrm{T}_{\mathrm{a}}=50.4 \mathrm{~K}\right)$ is about five times higher than that of IN939 $\left(\Delta \mathrm{T}_{\mathrm{a}}=10.1\right.$ $\mathrm{K})$. This difference in undercoolability can only be attributed to the chemical compositions of the used alloys, as the process conditions were the same. In alloy IN 939, the added alloying elements amount to about 50\% in weight, while in CMSX-6, alloying elements are only about $30 \%$ in weight. Obviously, it is the great difference in the amount of added alloying elements that results in the significant difference in undercoolability between the investigated alloys. The influence of individual alloying elements on the undercoolability should be determined in the future by investigating a great amount of superalloys.

Table 2. Measured undercooling values in the samples of IN939 and CMSX6

\begin{tabular}{|c|c|c|c|c|c|c|c|c|c|c|c|}
\hline \multirow{2}{*}{ Alloy } & \multicolumn{10}{|c|}{ Nucleation undercooling $\Delta \mathrm{T}_{\mathrm{N}}[\mathrm{K}]$} & \multirow{2}{*}{$\begin{array}{c}\text { Undercoolability } \\
\qquad \Delta \mathrm{T}_{\mathrm{a}}[\mathrm{K}]\end{array}$} \\
\hline & \multicolumn{5}{|c|}{ Cycle I } & \multicolumn{5}{|c|}{ Cycle II } & \\
\hline IN 939 & 5.5 & 10.8 & 8.6 & 10.9 & 5.7 & 7.4 & 17.2 & 12.4 & 12.7 & 9.9 & 10.1 \\
\hline CMSX-6 & 75.0 & 36.0 & 51.0 & 37.0 & $-*$ & 76.0 & 44.0 & 52.0 & 32.0 & $-*$ & 50.4 \\
\hline
\end{tabular}

(*One of the thermal couples was defective and did not provide useful data.)

In addition, it is also found that the undercooling values of both alloys measured in Cycle II are generally greater than those in the first cycle. This may be attributed to the cleaning effect of the overheating cycles. 


\subsection{Stray grain in SC samples}

Fig. 5 shows the typical macrostructure of the etched components of both alloys used in this research. The frames in the pictures indicate the locations of the observed stray grains. The CMSX6 samples reveal a prominent low tendency for stray grains. No stray grain were observed in either Geometry A or B of the CMSX-6 samples. In contrast, the platforms of IN 939 samples were very prone to stray grain formation, which appeared on all platform extremities of Geometry A samples. Even in the components of Geometry B, where the cross-sectional transition is less abrupt, the most platform extremities were also occupied by stray grains. The stray grains started at the extremities, where the thermal undercooling was the most distinct.

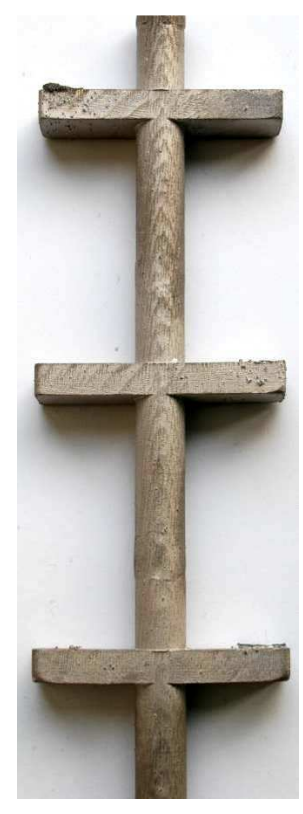

Geometry A

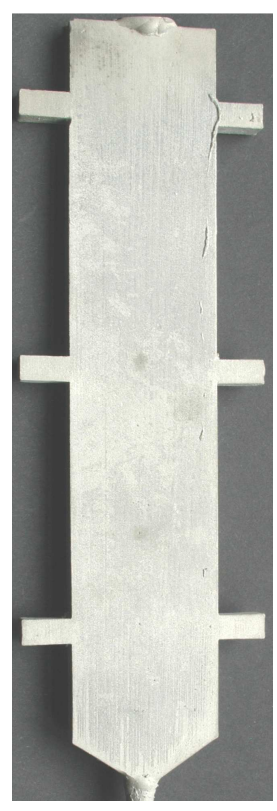

Geometry B

(a) CMSX-6

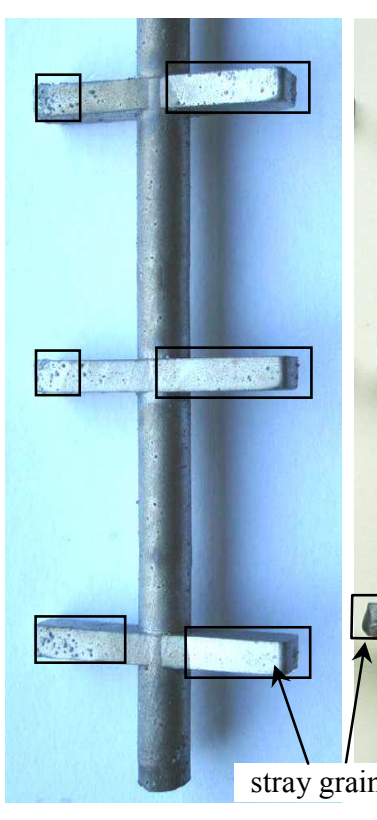

Geometry A
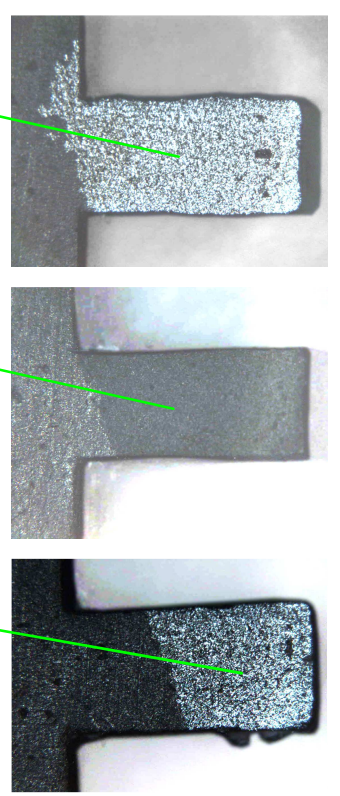

Geometry B

(b) IN 939

Fig.5. Typical macrostructure of the casting samples: (a) The samples of CMSX-6 are free of stray grains; (b) Each platform of Geometry A and most of Geometry B samples of IN 939 are captured by stray grains, which can be better identified in magnified photos on the right side .

It should be pointed out that the right side of the samples was the side facing the central rod in the shell mould cluster. On this side, a markedly higher tendency to stray grain formation can be observed in the IN 939 components, due to the significantly worse heating and cooling condition compared to the left side facing the heaters during DS process. For CMSX-6, however, both sides of the components are defect free.

\subsection{Dependence of stray grain formation on the undercoolability}

These stray grains were caused by a macroscopic curvature of the liquidus isotherm while it was passing through extreme enlargements in the cross-section of the component. As shown in Fig.6, a concave shaped liquidus isotherm exists in the platforms. Therefore an undercooled zone is formed at the extremities before the solidification front advances slowly into the platform. IN 939 has a very low undercoolability of only $10.1 \mathrm{~K}$, which can be easily exceeded by the local undercooling at the platform extremities, as shown in Fig.6a. In this case, an undesired nucleation of new grains is hardly avoidable.

By contrast, CMSX 6 is highly undercoolable. At the platform extremities of the CMSX-6 components the melt can be undercooled deeply without exceeding its undercoolability, until the primary crystal grows into the platform and then expands laterally towards the far corners of the platform (Fig.6b). In this case, the formation of stray grains is avoided. 


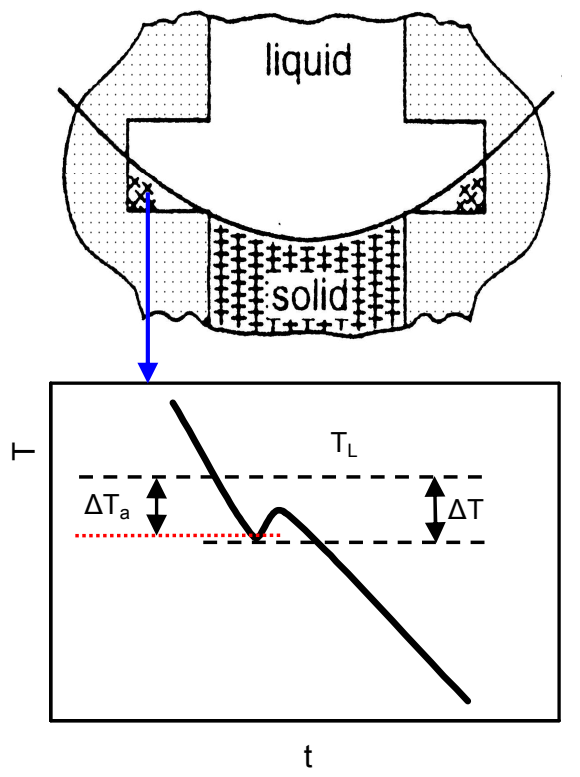

(a)

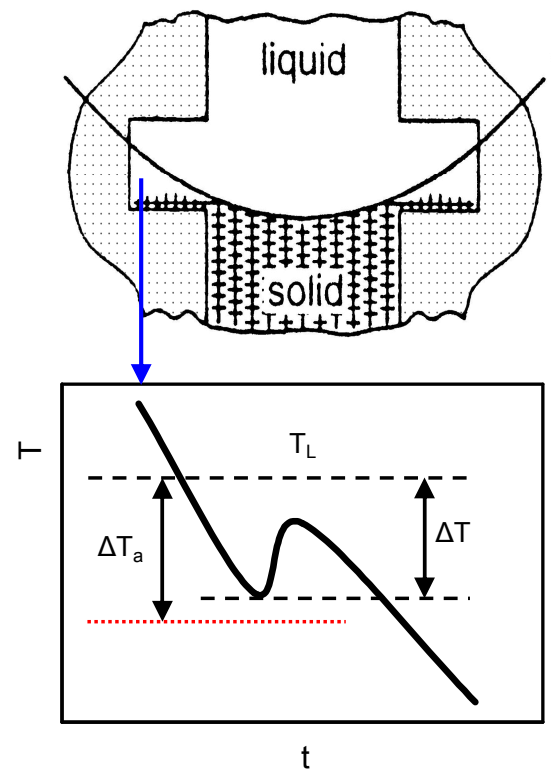

(b)

Fig.6. Schematic of the grain growth at the platform extremities depending on the undercoolability of the alloys: (a) For IN 939, the undercoolability of only $10.1 \mathrm{~K}$ can be easily exceeded by the local undercooling $\left(\Delta \mathrm{T}>\Delta \mathrm{T}_{\mathrm{a}}\right)$, resulting in the stray grain formation; (b) For CMSX-6, the melt can be undercooled deeply without exceeding its undercoolability $\left(\Delta \mathrm{T}<\Delta \mathrm{T}_{\mathrm{a}}\right)$, until the primary single crystal grows into the platform.

\section{Summary}

In the present work, the undercooling behaviour of the superalloys IN 939 and CMSX-6 during the isothermal heating and cooling processes was investigated. In the same shell mould and under the same process conditions the used alloys exhibited very different nucleation undercoolings. The undercoolability of CMSX- 6 was measured to be $50.4 \mathrm{~K}$ while that of IN 939 was only $10.1 \mathrm{~K}$. This great difference is assumed to be attributed to the amount of the added alloying elements. The repeating times of the heating-cooling cycles seems to also influence the measured value of nucleation undercooling.

Both alloys were directionally solidified with the same process parameters to produce simplified turbine blades. No stray grains were observed in the CMSX-6 samples due to its high undercoolability. By contrast, most platform extremities of IN 939 components were occupied by stray grains, because its low undercoolability could be easily exceeded by local undercooling. Thus, the difference of the superalloys in the tendency to form stray grains can be attributed to the difference in their undercoolability. It is believed that cast quality of SC components can be effectively improved by increasing the undercoolability of the superalloys.

\section{References}

[1] B.B. Seth: Superalloys 2000, edited by T.M. Pollock et al., TMS (2000), p. 3.

[2] M. Meyer ter Vehn, D. Dedecke, U. Paul and P.R. Sahm: Superalloys 1996, edited by R.D. Kissinger et al., TMS, (1996), p. 471.

[3] D. Ma and A. Bührig-Polaczek: Metal. Mater. Trans. B 40, (2009), p. 738.

[4] B. Lux, G. Haour and F. Mollard: Metallurgy, 35 (1981), p. 1235.

[5] I.A. Wagner and P.R. Sahm: Superalloys 1996, edited by. R.D. Kissinger et al., TMS, (1996) p. 497. 